AperTO - Archivio Istituzionale Open Access dell'Università di Torino

\title{
Ablation of Accessory Pathways with Uncommon Electrophysiologic Properties
}

\section{This is a pre print version of the following article:}

Original Citation:

\section{Availability:}

This version is available http://hdl.handle.net/2318/1769848

since 2021-01-28T15:23:30Z

Published version:

DOI:10.1016/j.ccep.2020.08.005

Terms of use:

Open Access

Anyone can freely access the full text of works made available as "Open Access". Works made available under a Creative Commons license can be used according to the terms and conditions of said license. Use of all other works requires consent of the right holder (author or publisher) if not exempted from copyright protection by the applicable law. 


\section{Ablation of accessory pathways with uncommon electrophysiologic properties}

Mario Matta ${ }^{1} \mathrm{MD}$, Jacopo Marazzato ${ }^{2,3} \mathrm{MD}$, Roberto De Ponti ${ }^{2,3} \mathrm{MD}$, Fiorenzo Gaita ${ }^{4} \mathrm{MD}$, Matteo Anselmino*5.

1.Cardiology Division, Sant'Andrea Hospital, Street address 13100 Vercelli, Italy; 2.Department of Heart and Vessels, Ospedale di Circolo, Viale Borri, 57, 21100 Varese, Italy; 3.Department of Medicine and Surgery, University of Insubria, Viale Guicciardini, 9, 21100 Varese, Italy; 4. Cardiovascular Department, Policlinico di Monza, Clinica Pinna Pintor, Street address CAP Turin, Italy; 5. Cardiology Division, Department of Medical Sciences, "Città della Salute e della Scienza di Torino" Hospital, University of Turin, Street address CAP Italy.

Conflicts of interest disclosure: Dr. De Ponti has received lecture fees from Biosense Webster and Biotronik, educational grants from Biosense Webster, Biotronik, Medtronic, Abbott, Boston Scientific, Bayer, Pfizer, Boehringer Ingelheim and research grants from Actelion. None for the other authors.

No funding sources to be acknowledged

\section{Word count: 5465}

E-mail addresses: mail Matta j.marazzato88@gmail.com; roberto.deponti@uninsubria.it; mail Gaita matteo.anselmino@unito.it

*Corresponding author:

Matteo Anselmino, MD

Cardiology Division, Department of Medical Sciences,

"Città della Salute e della Scienza di Torino" Hospital,

University of Turin,

Street address

CAP Turin, Italy

Phone: +39011

Fax: +39011

Email:matteo.anselmino@unito.it 
KEY-WORDS: catheter ablation, accessory pathways, atrio-ventricular fibers, atrio-fascicular fibers, nodoventricular fibers, nodo-fascicular fibers, fasciculo-ventricular fibers, permanent junctional reciprocating tachycardia.

\section{KEY POINTS:}

- Catheter ablation of accessory pathways is in general highly safe and effective, but in some cases uncommon bundles with node like conduction properties might pose challenges for proper diagnosis and successful ablation.

- Atrio-ventricular, atrio-fascicular, nodo-ventricular, nodo-fascicular, and fasciculo-ventricular fibers are to be included among accessory pathways with slow and decremental conduction properties.

- Permanent junctional reciprocating tachycardia (so-called Coumel tachycardia) is sustained by fibers with slow and decremental conduction properties, which are responsible for the peculiar characteristics to this arrhythmia.

- Knowledge of these variants of accessory pathway is paramount to warrant a safe and effective ablation in this complex clinical scenario.

\section{SYNOPSIS:}

In rare cases, atrioventricular re-entrant arrhythmias are sustained by accessory pathways with peculiar electrophysiologic features that may be related to their specific anatomy. Most of these bundles show decremental node like conduction properties and sustain peculiar forms of arrhythmias which require careful differential diagnosis. On the other hand, some pathways do not actively sustain any re-entrant circuit and should nevertheless be promptly recognised to avoid unnecessary ablation attempts. Although rare, these variants of accessory pathway should be known to warrant a safe and effective catheter ablation procedure. 


\section{Introduction}

As previously stated ${ }^{1,2}$, terms like "Mahaim fiber", "pseudo-Mahaim fibers", or "accessory pathways (AP) with Mahaim properties" should be avoided due to specific embryogenetic reasons and peculiar pathophysiological features of these fibers. A classification on anatomic grounds seems more appropriate in the setting, as proposed elsewhere in this issue, and it could be as follows:

A. Atrio-fascicular (AF) and atrio-ventricular (AV) pathways with node like conduction properties connecting the right atrium to the right bundle branch and the right ventricle, respectively.

B. Nodo-fascicular (NF) and nodo-ventricular (NV) pathways connecting the AV node to the right bundle branch and the right ventricle, respectively.

C. Fasciculo-ventricular AP connecting the His-Purkinje system to the upper right ventricular septum.

Fibers sustaining permanent junctional reciprocating tachycardias (PJRT), the so-called "Coumel tachycardia" should be also included among fibers with slow and decremental conduction properties.

The anatomy and pathophysiology of these AP variants and the related arrhythmia characteristics are described in a different section of this issue. Due to their peculiar electrophysiological properties, these uncommon fibers are approached in a distinct manner compared to the more common Kent bundles, when catheter ablation $(C A)$ is deemed necessary. The reported anatomic classification is relevant for a deep understanding of the tachycardia mechanism and, therefore, the pinpoint identification of the CA target in this complex clinical scenario. A summary of the characteristics of these AP variants and the criteria for differential diagnosis, together with the target and the results of CA are reported in the next paragraphs.

\section{Atrio-fascicular and atrio-ventricular accessory pathways}

\subsection{Electrocardiographic and electrophysiological features}

These AP responsible of variants of ventricular pre-excitation show the following characteristics at noninvasive and invasive evaluation:

\section{A. Minor or absent ventricular pre-excitation in sinus rhythm on surface $E C G^{3}$}

B. Evidence of slow, decremental, and node like conduction properties at electrophysiology study ${ }^{4,5}$. During programmed atrial stimulation (Figure 1), a progressive A-H interval prolongation and shortening of the $\mathrm{H}$ $\mathrm{V} /$ delta interval are commonly observed on the His-bundle catheter recording with concomitant increase of the degree of ventricular pre-excitation on surface ECG. Differently from Kent bundles, characterized by a rate-independent non-decremental conduction, a progressive prolongation of the A-delta interval with progressive shortening of coupling interval of atrial extrastimuli is observed.

C. Ability to sustain only antidromic atrio-ventricular re-entrant tachycardia with a left bundle branch block morphology ${ }^{3}$ Although some exceptional demonstration of ventriculo-atrial conduction has been provided ${ }^{6,7}$, lack of retrograde conduction allows these AP to sustain only antidromic AV re-entrant tachycardia (Figure 2).

D. Reverse activation of the His-Purkinje system during antidromic atrio-ventricular re-entrant tachycardia or maximally pre-excited beats ${ }^{4}$. In these conditions, the right bundle electrogram precedes the His bundle one, demonstrating retrograde involvement of the His/right bundle in these circumstances. 
E. Confirmation of pathway involvement in the tachycardia circuit and distant location from the AV node ${ }^{4,8,9}$. During tachycardia, late atrial extrastimuli delivered afar from the AV node area usually advance the following ventricular complex without advancing the atrial electrogram recorded from the His-bundle region, therefore resetting the tachycardia circuit.

\subsection{Catheter ablation}

\subsubsection{Target for ablation.}

Although with some exceptions ${ }^{10}$, a sharp and high frequency pathway potential is usually recorded at the antero-lateral region of the tricuspid annulus in most patients, ${ }^{8,11-13}$ as shown on Figure 1. Due to the slow conduction properties and the length of these fibers, a short A-V interval with fusion of the local atrial and ventricular bipolar electrogram is rarely seen at the site of the AP location (Figure 3 ). In case of AF or long $A V$ fibers, the pathway potential may be recorded remote from the tricuspid annulus and close to the right ventricular apex (Figure 4). If no pathway potential is recorded along the presumptive course of the fiber, identification of its distal insertion site in the right ventricle can guide $C A^{9,14}$. This site is generally identified by the earliest local bipolar ventricular activation preceding the ECG delta wave with a concomitant steep QS complex morphology in the unipolar recording. Pacemapping reproducing the same QRS complex morphology observed during tachycardia or maximal pre-excited beats has been reported to be useful in this setting ${ }^{14}$.

\subsubsection{Ablation endpoint.}

CA can be performed both during sinus rhythm or antidromic $\mathrm{AV}$ re-entrant tachycardia resulting in disappearance of ventricular pre-excitation or tachycardia interruption in the anterograde limb of the circuit, respectively. Since AV/AF fibers contain accessory AV nodal tissue, heat-induced automaticity during RF CA is commonly observed (Figure 5) during energy delivery ${ }^{15}$. Complete abolition of the conduction the AP is essential to avoid early or late arrhythmia recurrences.

\subsubsection{Ablation results and indications.}

Table 1 displays the efficacy, safety, and procedure data of CA of AF/AV pathways reported in 11 studies extracted from the available literature ${ }^{8-10,12,13,16-21}$. In the reported studies, 173 patients underwent CA targeting the pathway potential and/or its distal insertion site leading to interruption of clinical arrhythmia and/or disappearance of ventricular pre-excitation on surface ECG. Although the significant procedure time and the remarkable radiation exposure would prove the complexity of the procedure in some cases, CA of AF/AV pathways should be deemed highly safe and acutely effective. Moreover, CA using the reported ablation targets warrants the absence of arrhythmic recurrence at mid- and long-term followup after an initial successful procedure in most cases. Therefore, CA of AF/AV fibers is recommended for all patients with recurrent symptomatic tachycardia ${ }^{22}$, however, differently from what has been observed in asymptomatic patients with Kent bundles ${ }^{22}$, current guidelines do not recommend prophylactic ablation for prognostic reasons considering the exceedingly rare risk of sudden cardiac death occurring in these patients due to the peculiar electrophysiological properties of these fibers ${ }^{23}$.

In this setting, CA is usually performed using radiofrequency energy (RF) delivered by means of 4$\mathrm{mm}$ tip irrigated or non-irrigated catheters according to the anatomic site of the pathway. In case of close 
location to the $\mathrm{AV}$ node or His-bundle region, similarly to $\mathrm{CA}$ of para-Hisian $\mathrm{AP}^{24}$, cryoablation may be used to minimize the risk of $\mathrm{AV}$ block ${ }^{25}$ due to the well-known biophysical and histopathologic effects of this energy source $24,26,27$.

\subsubsection{Peculiar cases.}

Since embryogenic remnants of AV nodal tissue can be only found along the tricuspid annulus ${ }^{28}, A F$ and $A V$ pathways are commonly located in the right atrial chamber. However, left-sided decremental node like pathways have also been described ${ }^{29-33}$ In these cases, slow conduction may have also another pathophysiologic explanation. Ageing ${ }^{34}$ might partially explain the deterioration of electrical conduction over left-sided Kent bundles leading to slow conduction. Moreover, CA-induced modification of the electrical conduction during a partially successful procedure might as well play a role in slowing conduction over an AV AP ${ }^{35}$.

As an example of complex CA of left-sided AP with decremental conduction properties, we report the case of a 70-year-old patient referring for palpitation at the Center of Turin. Upon admittance, 12-lead ECG showed a wide QRS complex tachycardia with right bundle branch block morphology and inferior axis deviation (Figure 6 A). Sinus rhythm was only restored after electrical cardioversion with evidence of manifest ventricular pre-excitation consistent with an AP with right anteroseptal location (Figure $6 \mathbf{B}$ ). However, quite interestingly, adenosine test showed the same QRS complex morphology recorded during tachycardia (Figure 7). These findings would suggest the presence of two different AP and, hence, an electrophysiology procedure was performed. Programmed ventricular stimulation only showed retrograde conduction over the His-Purkinje system, while incremental atrial pacing resulted in the progressive lengthening of the A-delta interval and concomitant widening of the QRS complex on surface ECG: these findings were consistent with antegrade conduction over a right antero-septal pathway with slow and decremental conduction properties (Figure $8 \mathbf{A}$ ). Interestingly, atrial cycle lengths shorter than $370 \mathrm{~ms}$ resulted in a change of the QRS complex morphology, which now was identical to the one recorded during tachycardia and consistent with a left-sided location of another AP (Figure $8 \mathbf{B}$ ). Programmed atrial stimulation confirmed that the left AP showed slightly better conduction properties compared to right one (Figure 9). For this reason, the left-sided AP was initially targeted for CA. After unfruitful mapping at the lateral mitral annulus, a sharp pathway potential consistent with pathway location identification was found at the anteroseptal mitral annular region (Figure 10). At this site, quite surprisingly, RF delivery resulted in disappearance of both the two pre-excitation morphologies. Final incremental pacing from multiple atrial sites ruled out presence of residual ventricular pre-excitation.

This case is consistent with a decremental AP with a single atrial insertion site at the exceedingly rare location of the aortomitral continuity and two distinct ventricular insertions at the right ventricular septum and antero-lateral mitral annular region, respectively. The electrophysiology study and response to CA provided invaluable information for the understanding of the complex arrhythmogenic substrate leading to a definitive treatment in this patient.

\section{Nodo-fascicular and nodo-ventricular accessory pathways}

\subsection{Electrocardiographic and electrophysiological features}

These very rare AP show the following characteristics at non-invasive and invasive evaluation: 
B. Evidence of slow-conduction and decremental properties during electrophysiology study ${ }^{37}$. Like AF and AV pathways, incremental atrial pacing is associated with $\mathrm{A}-\mathrm{H}$ interval prolongation and shortening of $\mathrm{H}$ $\mathrm{V} /$ delta interval on His-bundle catheter with possibly progressive increase of ventricular pre-excitation on surface ECG.

C. Capability of both antegrade ${ }^{5}$ and retrograde conduction properties ${ }^{36,38}$. During antidromic re-entrant tachycardia, the morphology of the QRS complex varies according to the distal insertion site of the fibers, ranging from a typical left bundle branch block morphology to a minimal degree of ventricular preexcitation. As expected with an antidromic tachycardia, the His bundle is retrogradely activated from distal to proximal following the onset of the pre-excited QRS complex. Conversely, in case of fibers with concealed conduction, the QRS complex morphology during orthodromic re-entrant tachycardia usually replicates the one observed during sinus rhythm and tachycardia is generally reset or terminated by Hissynchronous premature ventricular complexes, proving evidence of the presence and the participation of an AP in the arrhythmia circuit ${ }^{38}$

D. Exclusion of an extranodal location. Differently from AF and AV pathways, the introduction of premature atrial beats at progressively shorter coupling intervals is not associated with the advance in the timing of the next ventricular complex during antidromic tachycardia ${ }^{11,39}$ with no change in in the $\mathrm{H}-\mathrm{H}$ or $\mathrm{V}-\mathrm{V}$ intervals ${ }^{39}$. These observations would support that the atria do not participate in the tachycardia circuit in arrhythmic substrates sustained by NF/NV fibers.

E. Ventriculo-atrial dissociation during tachycardia ${ }^{38}$. Since the atria are not part of the arrhythmic circuit, atrial activation might be overtaken by sinus rhythm. The observation of this rare phenomenon further rules out the presence of extranodal AP.

\subsection{Catheter ablation}

Due to the absence ${ }^{11}$ or the inconstant recording of a pathway potential ${ }^{36,38}, \mathrm{NV}$ and NF pathways can be targeted during $\mathrm{CA}$, as follows:

A. At the atrial insertion site in case of concealed pathways sustaining orthodromic re-entrant taachycardia. The earliest retrograde atrial activation is usually located at the mid-septal region ${ }^{36}$ or at the roof of the coronary sinus ostium ${ }^{38}$

B. Empirical ablation of the slow pathway ${ }^{40}$ in all other cases.

In this setting, due to the proximity of the AP to the AV node and His bundle region, cryoablation can be useful as an alternative energy source to avoid the risk of iatrogenic permanent AV block.

\section{Fasciculo-ventricular accessory pathways}

\subsection{Electrocardiographic and electrophysiologic features}

These AP, possibly observed in clinical practice, show the following characteristics at non-invasive and invasive evaluation: 
A. Minimal pre-excitation at baseline on surface $E C G^{37,41}$ usually consistent with a right anteroseptal or para-Hisian AP is present

B. Induction of complete AV block by bolus intravenous injection of adenosine ${ }^{34}$. Since the proximal insertion of this fibers is distal to the AV node/His bundle and, hence, dependent on nodal conduction, adenosine can create transient AV block with no modification of the degree of ventricular pre-excitation before and after block.

C. Unaltered ventricular pre-excitation during programmed atrial stimulation ${ }^{37,41}$. For the same reason, progressive prolongation of the $\mathrm{A}-\mathrm{H}$ and $\mathrm{A}$-delta intervals with no change in the $\mathrm{H}$-delta interval and the degree of ventricular pre-excitation is observed during programmed atrial pacing. This feature enables to differentiate $\mathrm{FV}$ fibers from Kent bundles, which are characterized by rate-independent non-decremental conduction, and from AF/AV and NF/NV decremental pathways, where the degree of pre-excitation changes at different cycle lengths. However, during programmed atrial stimulation with progressively shorter coupling intervals may cause disappearance of ventricular pre-excitation with narrowing of the QRS complex associated with the sudden prolongation of the $\mathrm{H}-\mathrm{V}$ interval, due conduction block over the FV fibers ${ }^{41}$ as shown in Figure 11A-B.

D. Inability to sustain re-entrant arrhythmias ${ }^{1,37,41}$. Due to the anatomic location immediately distal to the AV node/His bundle, FV fibers can only act as bystander fibers in a variety of arrhythmogenic substrates.

\subsection{Catheter ablation.}

Considering their peculiar anatomic location and electrophysiologic properties ${ }^{37,41}$, although with some exceedingly rare exceptions ${ }^{42,43}$, FV pathways do not usually sustain re-entrant arrhythmias ${ }^{41}$. Therefore, the role of CA is far less established in these patients and any pointless CA attempt may lead to AV node conduction damage, especially in young patients ${ }^{44}$.

\section{Concealed slow conducting accessory pathways sustaining permanent junctional reciprocating tachycardia}

\subsection{Electrocardiographic and electrophysiologic features}

The characteristics of these AP sustaining the PJRT or so-called "Coumel tachycardia" and usually located in the postero-medial right or left areas are as follows:

A. Concealed and decremental conduction properties. Normal P-R interval and no signs of overt ventricular pre-excitation in sinus rhythm on surface ECG with evidence of retrograde conduction during electrophysiology study is observed in these patients ${ }^{45,46}$. Decremental conduction properties are defined as an increment of more than $\mathbf{5 0}$ milliseconds of the ventriculo-atrial conduction time during programmed ventricular stimulation ${ }^{45}$.

B. Ability to sustain permanent junctional reciprocating tachycardia. PJRT is an orthodromic AVRT which displays a narrow QRS complex morphology, 1:1 AV relationship, a prolonged R-P'/ $P^{\prime}-R$ ratio with negative $P$ wave morphology in the inferior leads ${ }^{45,46}$, and an iterative clinical manifestation (Figure 12).

D. Confirmation of extranodal retrograde conduction during tachycardia. During tachycardia, delivery of His-refractory premature ventricular beats shows advancement (Figure 13) or paradoxical delay of the 
following retrograde atrial activation ${ }^{45,47}$, excluding atypical AV nodal re-entry or atrial tachycardia as potentially alternative arrhythmogenic mechanisms ${ }^{47}$. Of note, this modification in atrial activation is facilitated by ventricular pacing at a site close to the AP.

\subsection{Catheter ablation}

CA is highly safe and effective ${ }^{48}$ and strongly recommended in highly symptomatic patients or in cases with PJRT-related tachycardia-induced cardiomyopathy ${ }^{23}$. CA targets the earliest retrograde atrial activation during ongoing tachycardia ${ }^{47}$ and this anatomic location is commonly found at the posterior atrial septum close to the coronary sinus orifice ${ }^{45-47}$ (Figure 14 A) where an AP potential might be recorded ${ }^{49}$. Three-dimensional electroanatomic mapping systems may help to identify the proper CA site in this setting (Figure $14 B)^{50}$.

$\mathrm{CA}$ is generally performed using irrigated or non-irrigated RF catheters, however, cryoablation can be used as an alternative energy source to minimize the risk of AV block, especially in children and young adults $^{51}$.

\section{Conclusions}

Although rarely encountered in clinical practice, the electrophysiologist should be aware of these uncommon AP characterized by slow and decremental conduction properties to avoid troublesome procedures and potential damage to the His-Purkinje system especially in young patients. The reported anatomic classification, proper mapping and achievement of CA endpoints can warrant a safe and effective procedure in this peculiar clinical setting.

\section{References}

1. De Ponti R, Salerno-Uriarte JA. "Mahaim" fasciculoventricular fibers: Rare variant of ventricular preexcitation or subtle clinical problem? Heart Rhythm 2005;2:7-9.

2. Balaji S, Tchou P, Kanter R. Mahaim fibers: Should they be renamed? Heart Rhythm 2020;17:161-2.

3. Sternick EB, Timmermans $\mathrm{C}$, Sosa $\mathrm{E}$, et al. The electrocardiogram during sinus rhythm and tachycardia in patients with Mahaim fibers: The importance of an " $r$ " pattern in lead III. J Am Coll Cardiol 2004;44:1626-35.

4. Tchou P, Lehmann MH, Jazayeri M et al. Atriofascicular connection or a nodoventricular Mahaim fiber? Electrophysiologic elucidation of the pathway and associated reentrant circuit. Circulation 1988;77:837-48.

5. Klein GJ, Guiraudon GM, Kerr CR, et al. "Nodoventricular" accessory pathway: Evidence for a distinct accessory atrioventricular pathway with atrioventricular node-like properties. J Am Coll Cardiol 1988;11:1035-40.

6. De Ponti R, Storti C, Stanke A, et al. Ablazione transcatetere mediante radiofrequenza in pazienti con via anomala atrioventricolare destra a conduzione lenta tipo Mahaim [Radiofrequency catheter ablation in patients with Mahaim-type slow-conduction accessory right atrioventricular pathway]. Cardiologia 1994;39:169-80.

7. Kreiner G, Heinz G, Frey B et al. Demonstration of retrograde conduction over an atriofascicular accessory pathway. J Cardiovasc Electrophysiol 1997;8:74-9. 
8. McClelland JH, Wang X, Beckman KJ, et al. Radiofrequency catheter ablation of right atriofascicular (Mahaim) accessory pathways guided by accessory pathway activation potentials. Circulation 1994;89:2655-66.

9. Klein LS, Hackett FK, Zipes DP et al. Radiofrequency catheter ablation of Mahaim fibers at the tricuspid annulus. Circulation 1993;87:738-47.

10. Haghjoo M, Arya A, Emkanjoo Z et al. Is the activation potential of Mahaim pathway always a fast potential? Implication for radiofrequency catheter ablation. Europace 2005;7:440-6.

11. Grogin HR, Lee RJ, Kwasman M, et al. Radiofrequency catheter ablation of atriofascicular and nodoventricular Mahaim tracts. Circulation 1994;90:272-81.

12. Cappato R, Schlüter M, Weiß C, et al. Catheter-induced mechanical conduction block of right-sided accessory fibers with Mahaim-type preexcitation to guide radiofrequency ablation. Circulation 1994;90:282-90.

13. Brugada J, Martínez-Sánchez J, Kuzmicic B, et al. Radiofrequency catheter ablation of atriofascicular accessory pathways guided by discrete electrical potentials recorded at the tricuspid annulus. Pacing Clin Electrophysiol 1995;18:1388-94.

14. Ducceschi V, Vitale R, Ottaviano L et al. Ablating the ventricular insertion of atrio-fascicular Mahaim fiber: What selection criteria should we use? J Interv Card Electrophysiol 2009;25:207-11.

15. Sternick EB, Sosa EA, Timmermans C, et al. Automaticity in Mahaim fibers. J Cardiovasc Electrophysiol 2004;15:738-44.

16. Mönnig G, Wasmer K, Milberg P et al. Predictors of long-term success after catheter ablation of atriofascicular accessory pathways. Heart Rhythm 2012;9:704-8.

17. De Ponti R, Storti C, Salerno-Uriarte JA, et al. Tachicardia reciprocante atrioventricolare con QRS tipo blocco di branca sinistra in soggetti da sottoporre ad ablazione transcatetere mediante radiofrequenza: analisi del substrato e del meccanismo della tachicardia [Atrioventricular reciprocating tachycardia with QRS type left branch block in patients undergoing radiofrequency catheter ablation: analysis of the substrate and mechanism of tachycardia]. G Ital Cardiol 1994;24:707-21.

18. Heald SC, Davies DW, Ward DE et al. Radiofrequency catheter ablation of Mahaim tachycardia by targeting Mahaim potentials at the tricuspid annulus. Br Heart J 1995;73:250-7.

19. Silva MA, Berardi G, Kraemer A et al. Catheter ablation of atriofascicular Mahaim fibers guided by the activation potential. Arq Bras Cardiol 2003;80:61-70.

20. Kothari S, Gupta AK, Lokhandwala YY et al. Atriofascicular pathways: Where to ablate? Pacing Clin Electrophysiol 2006;29:1226-33.

21. Liao Z, Ma J, Hu J et al. New observation of electrocardiogram during sinus rhythm on the atriofascicular and decremental atrioventricular pathways: Terminal quantronic resonance system complex slurring or notching. Circ Arrhythmia Electrophysiol 2011;4:897-901.

22. De Ponti R, Marazzi R, Doni LA, et al. Invasive electrophysiological evaluation and ablation in patients with asymptomatic ventricular pre-excitation persistent at exercise stress test. Europace 2015;17:946-52.

23. Brugada J, Katritsis DG, Arbelo E et al. 2019 ESC Guidelines for the management of patients with supraventricular tachycardiaThe Task Force for the management of patients with supraventricular tachycardia of the European Society of Cardiology (ESC). Eur Heart J 2020;41:655-720. 
24. Marazzato J, Fonte G, Marazzi R et al. Efficacy and safety of cryoablation of para-Hisian and midseptal accessory pathways using a specific protocol: single-center experience in consecutive patients. J Interv Card Electrophysiol 2019;55:47-54.

25. Gaita F, Montefusco A, Riccardi R et al. Acute and long-term outcome of transvenous cryothermal catheter ablation of supraventricular arrhythmias involving the perinodal region. J Cardiovasc Med 2006;7:785-92.

26. Khairy $P$, Chauvet $P$, Lehmann J, et al. Lower incidence of thrombus formation with cryoenergy versus radiofrequency catheter ablation. Circulation 2003;107:2045-50.

27. De Ponti R. Cryothermal energy ablation of cardiac arrhythmias 2005: State of the art. Indian Pacing Electrophysiol J 2005;5:12-24.

28. Anderson RH, Sánchez-Quintana D, Mori S et al. Unusual variants of pre-excitation: From anatomy to ablation: Part I-Understanding the anatomy of the variants of ventricular pre-excitation. J Cardiovasc Electrophysiol 2019;30:2170-80.

29. Goldberger JJ, Pederson DN, Damle RS et al. Antidromic tachycardia utilizing decremental, latent accessory atrioventricular fibers: Differentiation from adenosine-sensitive ventricular tachycardia. J Am Coll Cardiol 1994;24:732-8.

30. Johnson CT, Brooks C, Jaramillo J et al. A left free-wall, decrementally conducting, atrioventricular (Mahaim) fiber: Diagnosis at electrophysiological study and radiofrequency catheter ablation guided by direct recording of a Mahaim potential. Pacing Clin Electrophysiol 1997;20:2486-8.

31. Tada H, Nogami A, Naito $S$ et al. Left posteroseptal mahaim fiber associated with marked longitudinal dissociation. Pacing Clin Electrophysiol 1999;22:1696-9.

32. Francia P, Pittalis MC, Ali $\mathrm{H}$ et al. Electrophysiological study and catheter ablation of a Mahaim fibre located at the mitral annulus-aorta junction. J Interv Card Electrophysiol 2008;23:153-7.

33. De Ponti R, Marazzato J, Bagliani G, et al. Peculiar Electrocardiographic Aspects of Wide QRS Complex Tachycardia: When Differential Diagnosis Is Difficult. Card Electrophysiol Clin 2018;10:31732.

34. De Ponti R, Marazzato J, Marazzi R, et al. Pre-eccitazione ventricolare in assenza di sintomi: che strada percorrere? [Asymptomatic ventricular pre-excitation: what management?]. G Ital Cardiol (Rome) 2018;19:161-9.

35. Sternick EB, Correa FS, Rego S et al. Postablation-acquired short atrioventricular Mahaim-type fibers: Observations on their clinical, electrocardiographic, and electrophysiologic profile. Heart Rhythm 2012;9:850-8.

36. Hluchy J, Schlegelmilch P, Schickel S et al. Radiofrequency ablation of a concealed nodoventricular Mahaim fiber guided by a discrete potential. J Cardiovasc Electrophysiol 1999;10:603-10.

37. Gallagher JJ, Smith WM, Kasell JH et al. Role of Mahaim fibers in cardiac arrhythmias in man. Circulation 1981;64:176-89.

38. Han FT, Riles EM, Badhwar N et al. Clinical Features and Sites of Ablation for Patients With Incessant Supraventricular Tachycardia From Concealed Nodofascicular and Nodoventricular Tachycardias. JACC Clin Electrophysiol 2017;3:1547-56.

39. Papagiannis J, Vachtsevanos L, Rammos S et al. Cryoablation of a nodoventricular Mahaim fiber. J Interv Card Electrophysiol 2005;14:111-6. 
40. Hoffmayer KS, Lee BK, Vedantham $V$ et al. Variable clinical features and ablation of manifest nodofascicular/ventricular pathways. Circ Arrhythmia Electrophysiol 2015;8:117-27.

41. Sternick EB, Gerken LM, Vrandecic MO et al. Fasciculoventricular Pathways: Clinical and Electrophysiologic Characteristics of a Variant of Preexcitation. J Cardiovasc Electrophysiol 2003;14:1057-63.

42. Suzuki F, Okishige $\mathrm{K}$, Hirao $\mathrm{K}$ et al. Electrocardiographic masking of a myocardial infarction by a fasciculoventricular Mahaim fiber, atrioventricular reentry with anterograde conduction over a fasciculoventricular Mahaim fiber and retrograde conduction over a concealed Kent bundle. Jpn Heart J 1986;27:759-69.

43. Cao K, Chen M, Zou J et al. Narrow QRS tachycardia with ventriculoatrial dissociation mediated by a left fasciculoventricular fiber. J Interv Card Electrophysiol 2005;13:151-7.

44. Kim YG, Nam GB, Cho MS et al. Impact of fasciculoventricular bypass tracts on the diagnosis and treatment of concomitant arrhythmias and cardiac diseases. J Electrocardiol 2019;55:34-40.

45. Critelli G, Gallagher JJ, Monda V et al. Anatomic and electrophysiologic substrate of the permanent form of junctional reciprocating tachycardia. J Am Coll Cardiol 1984;4:601-10.

46. Guarnieri T, Sealy WC, Kasell JH et al. The nonpharmacologic management of the permanent form of junctional reciprocating tachycardia. Circulation. 1984;69:269-77.

47. Gaita F, Haissaguerre M, Giustetto $C$ et al. Catheter ablation of permanent junctional reciprocating tachycardia with radiofrequency current. J Am Coll Cardiol 1995;25:648-54.

48. Kang KT, Potts JE, Radbill AE, et al. Permanent junctional reciprocating tachycardia in children: a multicenter experience. Heart Rhythm 2014;11:1426-32.

49. Scaglione $M$, Caponi D, Riccardi R et al. Accessory pathway potential recording in a case of permanent junctional reciprocating tachycardia with decremental conduction localized on the atrial site. Ital Heart J 2001;2:147-51.

50. Scaglione M, Ebrille $E$, Caponi D et al. Zero-fluoroscopy ablation of accessory pathways in children and adolescents: CARTO3 electroanatomic mapping combined with RF and cryoenergy. Pacing Clin Electrophysiol 2015;38:675-81.

51. Gaita F, Montefusco A, Riccardi R et al. Cryoenergy catheter ablation: a new technique for treatment of permanent junctional reciprocating tachycardia in children. J Cardiovasc Electrophysiol 2004;15:263-8. 


\section{Figure Legend.}

Figure 1. Slow-conductive and decremental properties of an atrio-ventricular node like accessory pathway. From top to bottom, bipolar signals recorded from diagnostic catheters positioned at the high right atrium (HRA), His-bundle region ( $\mathrm{HBE}$ ), and coronary sinus (CS) are displayed. Electrograms recorded by the ablation catheter located on the accessory pathway $(A B L)$, with the corresponding unipolar recording ( $A B L$ $U)$, and surface ECG leads are also shown. A sharp pathway potential $(M)$ is consistently recorded on the ablation catheter. The A-M interval is expression of conduction over the proximal part of the accessory bundle and its decremental properties are proved by the prolongation of $\mathrm{A}-\mathrm{M}$ and $\mathrm{A}-\mathrm{V}$ interval during the paced beat from the HRA. Interestingly, compared to the A-H interval representing A-V node conduction, this accessory pathway shows faster conduction and less decremental properties in the premature atrial beat, leading to a greater degree of ventricular pre-excitation on surface ECG.

Figure 2. Antidromic atrioventricular re-entrant tachycardia with left bundle branch morphology sustained by atrioventricular fibers with node-like conduction properties.

Figure 3. Intracavitary recordings from the His bundle catheter (HIS p), coronary sinus catheter (CS), mapping/ablation catheter (ABL d bipolar recording; UNI unipolar recording), right ventricular catheter (RVA d) are displayed together with surface tracings. A sharp and high-frequency potential (so called Mahaim potential) is recorded between the atrial and ventricular deflections in $A B L d$, located onto atriofascicular fibers with node like conduction properties. The local atrial and ventricular potentials are clearly separated, and ventricular pre-excitation is present of surface ECG.

Figure 4.From top to bottom, the signal are displayed from the followings catheters: quadripolar catheter positioned at the His-bundle region from proximal (HBEp) to distal (HBEd), multipolar coronary sinus catheter (CS) from proximal to distal (from $\mathrm{CS}_{7,8}$ to $\mathrm{CS}_{1,2}$ ), bipolar recordings of an ablation catheter from distal ( $A B L d$ ) to proximal (ABLp), diagnostic catheter at the right ventricular apex (RVap), and surface ECG leads (I,II, III, and V1). During antidromic tachycardia, a sharp pathway potential (black arrows) is recorded $40 \mathrm{~ms}$ before the QRS onset at the ventricular insertion site of these atrio-fascicular fibers remote from the tricuspid annulus. The distal ventricular insertion is confirmed by absence of atrial electrogram preceding the pathway potential and the local ventricular electrogram.

Figure 5. Tachycardia interruption during radiofrequency energy delivery. Same catheter display as in Figure 4. After proper mapping, antegrade conduction over the pathway is abolished by radiofrequency energy delivery with interruption of the antidromic atrioventricular re-entrant tachycardia in the antegrade limb of the re-entry circuit. Upon interruption, the sinus beat shows no sign of ventricular pre-excitation. Of note, shortening of the tachycardia cycle consistent with heat-induced automaticity during ablation is transiently observed in the last tachycardia beat before its interruption.

Figure 6 A-B. Twelve-lead ECG showing a wide QRS complex tachycardia with right bundle branch block morphology and inferior axis deviation in a 70-year-old patient referring to the Emergency Department for palpitations (Panel A). After electrical cardioversion, sinus rhythm is restored, and surface ECG clearly shows a certain degree of ventricular pre-excitation consistent with a right antero-septal accessory pathway (Panel B).

Figure 7. Adenosine test in the same patient. After adenosine bolus injection, maximal ventricular preexcitation was evident during sinus rhythm with a QRS complex morphology in the peripheral leads identical to the one recorded during tachycardia. Interestingly, the last beat (arrow) shows a pre-excited 
beat similar to the one shown in sinus rhythm after electrical cardioversion. These findings suggest the presence of two different accessory pathways in this patient.

Figure 8 A-B. Programmed atrial stimulation during electrophysiology study in the same patient. Maximal degree of ventricular pre-excitation with a QRS complex morphology consistent with a right anteroseptal accessory pathway is evident during programmed atrial pacing (Panel A). A very different pre-excited QRS complex morphology consistent with a left-sided accessory pathway is clearly seen when atrial pacing is performed at cycle length shorter than $370 \mathrm{~ms}$ (Panel B) suggesting the presence of a right- and left-sided accessory pathway in this patient.

Figure 9. Incremental atrial pacing in the same patient shows the different electrophysiological properties of the two observed accessory pathways. Initially, antegrade conduction occurs over the right anteroseptal bundle, as suggested by the left bundle branch block-like morphology of the QRS complex but, as the atrial cycle length shortens, QRS complex morphology gradually changes and the last two beats (black arrows) shows a QRS complex morphology identical to the one recorded during tachycardia and consistent with a left-sided pathway.

Figure 10 A-B. Fast anatomical mapping in the same patient representing both atria performed by means of three-dimensional electroanatomic mapping systems using a contact-force sensing catheter. The yellow and the pink tags represent the area of the His-bundle and the successful ablation site, respectively. In the latter site, a sharp pathway potential (so-called Mahaim potential) was clearly recorded on the ablation catheter between the atrial and ventricular deflections shown in the lower right inset. $A B L=$ successful ablation site; $C S=$ coronary sinus catheter; $L A=$ left atrium; $M A=$ mitral annulus; $R A=$ right atrium; $\mathrm{TA}=$ tricuspid annulus.

Figure 11 A-B. Electrophysiological properties of a fasciculo-ventricular accessory pathway. In Panel A, during programmed atrial stimulation, the premature beat (third) shows the same degree of ventricular pre-excitation and a short $\mathrm{H}-\mathrm{V}$ interval as in the first two beats. In Panel B, a more premature atrial extrastimulus at $330 \mathrm{~ms}$ achieves the effective refractory period of the fasciculo-ventricular fibers leading to loss of ventricular pre-excitation and a normal $\mathrm{H}-\mathrm{V}$ interval.

Figure 12. Iterative form of permanent junctional reciprocating tachycardia. No atrial or ventricular premature beats are required for tachycardia onset. The incessant presentation of this arrhythmia is a peculiar diagnostic feature (see text for further explanation)

Figure 13. Decremental properties of fibers sustaining the so-called "Coumel tachycardia". From top to bottom, signals recorded from the following catheters are displayed: quadripolar catheter positioned at the His-bundle region from proximal (HBEp) to distal (HBEd), decapolar coronary sinus catheter (CS) from proximal to distal (from $\mathrm{CS}_{9,10}$ to $\mathrm{CS}_{1,2}$ ), ablation catheter (ABLp), and surface ECG leads (V1,I, and II). Hisrefractory premature ventricular beat advances the following atrial activation $(A)$ during ongoing tachycardia. Ventriculo-atrial (VA) interval measured after His-refractory premature ventricular beat is longer (348 ms) compared to the one recorded during tachycardia ( $305 \mathrm{~ms}$ ) confirming the decremental conduction properties of the concealed accessory pathway sustaining the permanent junctional reciprocating tachycardia.

Figure 14 A-B. Ablation of permanent junctional reciprocating tachycardia; tracings are as in the previous figure, with the addition of bipolar recording from the proximal electrode pair of the ablation catheter (ABL p). During tachycardia, the earliest retrograde atrial activation is recorded in the right postero-septal region 
and precedes by $63 \mathrm{~ms}$ the activation in the proximal coronary sinus (Panel A). In Panel B, fast anatomic mapping of the right atrium during tachycardia is displayed in left anterior oblique view: the earliest retrograde atrial activation close to the coronary sinus orifice is identified by the red area on this map. In this site, catheter ablation resulted in tachycardia interruption and suppression of conduction over the accessory pathway. 
Table 1.

Efficacy and safety of catheter ablation of atrio-fascicular and atrio-ventricular accessory pathways.

\begin{tabular}{|c|c|c|c|c|c|c|c|c|c|c|c|c|c|c|}
\hline Author & Year & Pop. $n$ & $\begin{array}{c}\text { Age } \\
\text { (years) }\end{array}$ & $\mathrm{AF} / \mathrm{AV}, \mathrm{n}$ & Ebstein, $n$ & $\begin{array}{c}\text { Concomitant } \\
\text { substrate }\end{array} \mid$ & $\begin{array}{c}\text { Acute } \\
\text { success } \\
\text { n, } \%\end{array}$ & $\begin{array}{c}\mathrm{RF} \\
\text { Ablations, } \mathrm{n}^{*}\end{array}$ & Complications & $\mathrm{FT}(\mathrm{min})$ & PT (min) & $\begin{array}{l}\text { Long-term } \\
\text { success } \\
\text { n, } \%\end{array}$ & Proc/pts & $\begin{array}{l}\text { Follow-up } \\
\text { (months) }^{*}\end{array}$ \\
\hline Klein ${ }^{9}$ & 1993 & 4 & 27 [11-48] & $3 / 1$ & 0 & AVNR & $\begin{array}{c}4 / 4 \\
(100 \%) \\
\end{array}$ & $15(10-19)$ & 0 & $\mathrm{~N} / \mathrm{A}$ & N/A & 4/4 (100\%) & $4 / 4$ & $8(2-15)$ \\
\hline De Ponti ${ }^{17}$ & 1994 & 3 & N/A & N/A & 2 & AVRT,AVNRT & $\begin{array}{c}3 / 3 \\
(100 \%) \\
\end{array}$ & $\mathrm{N} / \mathrm{A}$ & 0 & N/A & N/A & $3 / 3(100 \%)$ & $3 / 3$ & $8 \pm 7$ \\
\hline Cappato $^{12}$ & 1994 & 11 & $30 \pm 9$ & $3 / 9$ & 0 & N/A & $\begin{array}{c}9 / 11 \\
(81 \%) \\
\end{array}$ & $7 \pm 5$ & 0 & $86 \pm 37$ & $438 \pm 192$ & 8/11 (73\%) & $23 / 11$ & $9 \pm 2$ \\
\hline McClelland ${ }^{8}$ & 1994 & 23 & $30 \pm 10$ & $23 / 0$ & 1 & AVRT,AVNRT & $\begin{array}{c}23 / 23 \\
(100 \%)\end{array}$ & $3 \pm 3$ & 0 & $99 \pm 39$ & N/A & 23/23 (100\%) & $23 / 23$ & $18 \pm 13$ \\
\hline Heald $^{18}$ & 1995 & 19 & 33 [7-53] & N/A & 2 & AVRT,AVNRT & $\begin{array}{l}18 / 19 \\
(95 \%)\end{array}$ & 3 [1-13] & 0 & 30 [7-107] & 120 [49-260] & 17/19 (89\%) & $20 / 19$ & 9 [1-29] \\
\hline Brugada $^{13}$ & 1995 & 4 & 35 [27-47] & $4 / 0$ & 1 & AVRT & $\begin{array}{c}4 / 4 \\
(100 \%) \\
\end{array}$ & 7 [1-14] & 0 & N/A & $\mathrm{N} / \mathrm{A}$ & 4/4 (100\%) & $4 / 4$ & $5[3-9]$ \\
\hline Silva ${ }^{19}$ & 2003 & 6 & $26 \pm 7.3$ & $6 / 0$ & 0 & AVRT & $\begin{array}{c}6 / 6 \\
(100 \%) \\
\end{array}$ & $5 \pm 3$ & 0 & $47 \pm 25$ & $178.6 \pm 108$ & $6 / 6(100 \%)$ & $9 / 6$ & $20 \pm 4$ \\
\hline Haghjoo $^{10}$ & 2005 & 10 & $30 \pm 12$ & $10 / 0$ & 1 & AVRT & $\begin{array}{c}10 / 10 \\
(100 \%) \\
\end{array}$ & $3 \pm 1$ & 0 & $35 \pm 13$ & $81 \pm 25$ & $10 / 10(100 \%)$ & $10 / 10$ & $12 \pm 3$ \\
\hline Kothari ${ }^{20}$ & 2006 & 29 & $19 \pm 8$ & $29 / 0$ & 3 & AVRT, AVNRT & $\begin{array}{l}28 / 29 \\
(97 \%)\end{array}$ & $6[1-13]$ & 0 & $35 \pm 12$ & $150 \pm 32$ & $28 / 29(97 \%)$ & $31 / 29$ & $19 \pm 13$ \\
\hline Liao $^{21}$ & 2011 & 30 & $37 \pm 12$ & $10 / 20$ & 1 & N/A & $\begin{array}{c}30 / 30 \\
(100 \%) \\
\end{array}$ & $\mathrm{N} / \mathrm{A}$ & 0 & N/A & N/A & $30 / 30(100 \%)$ & $30 / 30$ & $17 \pm 4$ \\
\hline Mönnig ${ }^{16}$ & 2012 & 34 & $37 \pm 17$ & $34 / 0$ & 0 & N/A & $\begin{array}{l}23 / 34 \\
(68 \%)\end{array}$ & $\mathrm{N} / \mathrm{A}$ & 0 & N/A & $\mathrm{N} / \mathrm{A}$ & $20 / 34(60 \%)$ & $43 / 34$ & $112 \pm 66$ \\
\hline
\end{tabular}

* Numbers expressed as either mean values \pm standard deviation or median values and $25-75 \%$ interquartile range in square brackets. 
Abbreviations. AF=atrio-fascicular accessory pathway; $\mathrm{AV}=$ atrioventricular accessory pathway; $\mathrm{AVNR}=$ atrioventricular nodal re-entry; $\mathrm{AVNRT}=a$ atrioventricular nodal re-entrant tachycardia; AVRT= atrioventricular re-entrant tachycardia sustained by Kent bundles; Ebstein= concomitant presence of Ebstein disease; FT= fluoroscopy time; Proc/pts= total number of procedures/total number of patients; PT=procedure time; RF=radiofrequency energy. 